\title{
Neuroleptoanalgesia associada à anestesia epidural com lidocaína e xilazina em cutias (Dasyprocta aguti)
}

\author{
Neuroleptoanalgesic associated to epidural anesthesia with lidocaine and xylazine in agoutis \\ (Dasyprocta aguti)
Fernanda Tércia Silva Cardosoㄹ, Francisco Solano Feitosa Júnior ${ }^{2}$, Bruno Lendro Maranhão Diniz², Luciano Ursulino de Lucena ${ }^{3} \&$ José de Ribamar da Silva Júnior ${ }^{4}$

\begin{abstract}
RESUMO
A anestesia epidural tem sido utilizada, freqüentemente, pela possibilidade do emprego de doses menores de fármacos que as administradas por outras vias, com menores efeitos adversos e sua associação com a neuroleptoanalgesia pode garantir uma anestesia com profunda sedação e analgesia. Objetivou-se descrever a neuroleptoanalgesia associada à anestesia epidural com xilazina e lidocaína em cutias, utilizando-se seis animais escolhidos aleatoriamente entre machos e fêmeas. Os animais receberam como neuroleptoanalgesia, na mesma seringa fentanil $(0,02 \mathrm{mg} / \mathrm{Kg})$ e droperidol $(2 \mathrm{mg} / \mathrm{Kg})$ por via intramuscular. Após 25 minutos, foi feita a injeção epidural no espaço lombossacro com lidocaína ( $7 \mathrm{mg} / \mathrm{Kg})$ e xilazina (0,5mg/ $\mathrm{Kg}$ ) ambos na mesma seringa aplicados durante um minuto. Foram avaliados a freqüência cardíaca, frequiência respiratória, temperatura retal e saturação de oxigênio nos momentos: M0 (antes da neuroleptoanalgesia), M1 (25 minutos após a neuroleptoanalgesia), M2 (logo após a aplicação da anestesia epidural) e posteriormente a cada dez minutos até o M8 (sessenta minutos após a aplicação da epidural). A utilização de xilazina/lidocaína por via epidural pode ser utilizada para a realização de cirurgias pré-umbilicais; a utilização de fentanil associado ao droperidol nas doses utilizadas não foram suficientes para produzir sedação nos animais a ponto de realizar a anestesia epidural com tranqüilidade, sendo, então, necessários mais estudos a respeito do uso da neuroleptoanalgesia nesses animais.
\end{abstract}

Descritores: Fentanil, Droperidrol, Analgesia.

\begin{abstract}
The epidural anesthesia has been used, often, by the possibility of the use in smaller doses of drugs than in the others ways, with fewer adverse effects and their association with neuroleptoanalgesia can guarantee a deep anesthesia with sedation and analgesia. With the objective to describe the neuroleptoanalgesia associated with epidural anesthesia with xylazine and lidocaine in agoutis, wore used six animals chosen between males and females. The animals received as a neuroleptoanalgesia, in the same syringe fentanyl $(0.02 \mathrm{mg} / \mathrm{kg})$ and droperidol $(2 \mathrm{mg} / \mathrm{Kg}) \mathrm{IM}$. After 25 minutes the epidural anesthesia was injected in the lombossacro space with lidocaine $(7 \mathrm{mg} / \mathrm{Kg})$ and xylazine $(0.5 \mathrm{mg} / \mathrm{kg})$ both in the same syringe applied for one minute. Were evaluated heart rate, respiratory frequency, rectal temperature and oxygen saturation in moments: M0 (before neuroleptoanalgesia), M1 (25 minutes after the neuroleptoanalgesia), M2 (after the application of epidural anesthesia) and after every ten minutes until the moment M8 (sixty minutes after application of epidural). The use of xilazina/lidocaine in the epidural anesthesia can be used for surgeries in pre umbilical area; the use of fentanyl associated with droperidol in the doses used were not sufficient to produce sedation in the animals to hold the epidural anesthesia with tranquility, so its required more studies with the use of neuroleptoanalgesia in these animals.
\end{abstract}

Keywords: Fentanyl, Droperidrol, Analgesia.

${ }^{1}$ Mestranda, Programa de Pós-graduação em Ciência Animal, Universidade Federal do Piauí, Teresina, PI/Brasil. ${ }^{2}$ Departamento de Clínica e Cirurgia da Universidade Federal do Piauí. ${ }^{3}$ Parque Zoobotânico de Teresina. ${ }^{4}$ Departamento de Clínica e Cirurgia da Universidade Estadual do Maranhão, São Luís, MA/Brasil. CORRESPONDÊNCIA: F.T.S. Cardoso [fernandatercia@ hotmail.com]. 


\section{INTRODUÇÃO}

Apesar de ser uma técnica consagrada, a anestesia epidural tem sido utilizada, freqüentemente, pela possibilidade do emprego de doses menores de fármacos que as administradas por outras vias, com menores efeitos adversos, além de promover analgesia intra e pós-operatória [12] e permitir a redução da resposta ao estresse pós-cirúrgico [22].

O uso isolado de anestésico local, normalmente da lidocaína, não é suficiente para produzir anestesia que viabilize uma ovariosalpingohisterectomia [8]. Adicionalmente, a lidocaína é um fármaco de curta ação, sendo limitada em cirurgias prolongadas, havendo necessidade da associação com fármacos de longa duração [5,8].

O objetivo dessa pesquisa foi descrever a neuroleptoanalgesia associada à anestesia epidural com xilazina e lidocaína em cutias, criadas em cativeiro, proporcionando bases para futuros experimentos.

\section{MATERIAIS E MÉTODOS}

Após aprovação do Comitê de Ética para Pesquisa da Universidade Federal do Piauí, sob parecer $\mathrm{n}^{\circ}$ 0538/06 e de acordo com os princípios éticos recomendados pelo Colégio Brasileiro de Experimentação Animal (COBEA), foram utilizadas seis cutias adultas, escolhidas aleatoriamente entre machos e fêmeas, pesando entre 1,5 e 2,5 quilos.

Os animais foram provenientes do Núcleo de Estudos e Preservação de Animais Silvestres, do Centro de Ciências Agrárias da Universidade Federal do Piauí (UFPI), Campus Socopo, Teresina-PI e foram submetidos a jejum sólido de 12 horas e líquido de 8 horas e, antes do experimento, foram levados em gaiolas individuais ao hospital veterinário e lá permaneceram por 12 horas, passando por um período de adaptação.

As cutias foram contidas fisicamente em sacos de polipropileno e pesadas em seguida. Antes de iniciar o procedimento anestésico (momento $0-\mathrm{M} 0$ ) os animais tiveram a temperatura aferida através do reto, com termômetro digital; a freqüência respiratória foi avaliada pela observação dos movimentos respiratórios durante um minuto; a frequiência cardíaca avaliada a partir do eletrocardiograma (ECC) e a saturação de oxigênio mensurada através do oxímetro de pulso. Esses parâmetros foram anotados em fichas individuais.
Os animais foram submetidos a uma neuroleptoanalgesia com o uso de fentanil ${ }^{1}$ na dose de 0,02 $\mathrm{mg} / \mathrm{Kg}$ associado ao droperidrol ${ }^{2} 2 \mathrm{mg} / \mathrm{Kg}$ ambos aplicados na mesma seringa e após 25 minutos da aplicação todos os parâmetros avaliados no M0 foram coletados novamente.

Após a mensuração dos parâmetros no M1 (25 minutos após a neuroleptoanalgesia), o espaço lombossacro era localizado e feito tricotomia e assepsia da cutis com álcool iodado a $1 \% 3$. Posteriormente, os animais foram colocados em decúbito esternal, com os membros pélvicos tracionados cranialmente, para administração da injeção epidural no espaço lombosacro. Um botão anestésico foi feito no local da injeção, localizado por palpação das tuberosidades ilíacas. Uma agulha 25 x 7 foi introduzida cranial-mente em ângulo aproximado de 70 graus (entre a $7^{\circ}$ lombar e a primeira sacral), até ultrapassar o ligamento amarelo e atingir o espaço epidural, o qual foi confirmado pela aspiração de uma gota do anestésico, depositada no canhão da agulha. Em seguida, administrou-se lentamente cloridrato de lidocaína ${ }^{4}(7 \mathrm{mg} / \mathrm{Kg})$ associado a xilazina $^{5}(0,5 \mathrm{mg} / \mathrm{Kg})$ ambos aplicados na mesma seringa durante um minuto.

O parâmetro qualidade de sedação da neuroleptoanalgesia foi transformado em escores para avaliação da latência do anestésico de acordo com o quadro 1.

O oximetro de pulso foi colocado na cartilagem auricular do animal, permanecendo durante todo o período de avaliação. Os valores da saturação de oxigênio foram anotados em cada momento de observação.

A cada dez minutos era aferida a temperatura retal, saturação de oxigênio, freqüência cardíaca e frequiência respiratória. Os outros tempos avaliados foram M2 (logo após a aplicação da anestesia epi-dural) M3 (dez minutos após a aplicação da epidural), M4 (vinte minutos após a aplicação da epidural), M5 (trinta minutos após a aplicação da epidural), M6 (quarenta minutos após a aplicação da epidural), M7 (cinquenta minutos após a aplicação da epidural) e M8 (sessenta minutos após a aplicação da epidural).

Os animais foram avaliados por 60 minutos após a anestesia epidural, observando-se o grau de sensibilidade através do pinçamento do membro pélvico, do espaço interdigital e do abdome na região 
Quadro 1. Parâmetro para mensuração da variável qualidade de sedação em escores.

\begin{tabular}{|c|l|l|l|l|}
\hline \multirow{2}{*}{ Parâmetro } & \multicolumn{4}{|c|}{ Classificação } \\
\cline { 2 - 5 } & \multicolumn{1}{|c|}{0} & \multicolumn{1}{|c|}{2} & \multicolumn{1}{c|}{3} \\
\hline \multirow{2}{*}{$\begin{array}{c}\text { Qualidade de } \\
\text { sedação }\end{array}$} & $\begin{array}{l}\text { Sem sedação, } \\
\text { alerta e } \\
\text { totalmente } \\
\text { responsivo a } \\
\text { estímulos } \\
\text { externos }\end{array}$ & $\begin{array}{l}\text { Discreta sedação, } \\
\text { com algum grau } \\
\text { de ataxia, com } \\
\text { diminuição da } \\
\text { percepção dos } \\
\text { estímulos } \\
\text { externos e } \\
\text { discreto } \\
\text { abaixamento de } \\
\text { cabeça }\end{array}$ & $\begin{array}{l}\text { Moderada } \\
\text { sedação com } \\
\text { possibilidade de } \\
\text { decúbito esternal } \\
\text { ou constante } \\
\text { ataxia dos } \\
\text { membros } \\
\text { pélvicos com } \\
\text { mínima resposta } \\
\text { a estímulos } \\
\text { externos. }\end{array}$ & $\begin{array}{l}\text { Extremamente } \\
\text { sedado, } \\
\text { decúbito lateral } \\
\text { com } \\
\text { incapacidade de } \\
\text { manter a cabeça } \\
\text { na posição } \\
\text { normal. }\end{array}$ \\
\hline
\end{tabular}

Variável "qualidade de sedação" modificados de Carrol [3] e O’Hair et al. [14].

retro umbilical, aferido por meio de estímulo nociceptivo mecânico, com pinça hemostática Kelly imediatamente após a mensuração das variáveis paramétricas [18].

O período de recuperação (PR) ou tempo hábil de analgesia foi definido como o período entre a aplicação da injeção da anestesia epidural e o retorno dos primeiros reflexos podais, levantamento de cabeça e tentativa de ficar em posição quadrupedal. O período de posição quadrupedal (PQ) foi definido como o espaço de tempo compreendido entre o início da ação da anestesia epidural e a total habilidade para caminhar.

Os resultados foram avaliados através da análise de variância (ANOVA) para verificar a variação da freqüência cardíaca, freqüência respiratória, temperatura retal e saturação de oxigênio entre os animais anestesiados neste experimento. Foi aplicado o teste Student-Newman-Keuls (SNK), com nível de significância estipulados em 5\% ( $<<0,05)$ para comparação entre as médias, calculando-se o coeficiente de variação [19].

\section{RESULTADOS}

Não houve sedação nos animais após a aplicação da neuroleptoanalgesia nos animais com as doses administradas (Grau de sedação 0). Este fato dificultou a aplicação da anestesia epidural, já que os animais apresentaram-se alerta e responsivos aos estímulos externos.

$\mathrm{Na}$ avaliação dos momentos anestésicos para a frequência cardíaca, frequiência respiratória, temperatura retal e saturação de oxigênio; percebe-se que a frequência respiratória foi o único parâmetro que apresentou diferenças significativas entre os momentos avaliados, diferença esta observada nos momentos 0 e 1 (Tabela 1).

$O$ período de recuperação dos animais utilizados neste experimento foi de 51,66 $\pm 4,68$ minutos e o período de posição quadrupedal foi de 79,05 $\pm 17,61$ minutos.

\section{DISCUSSÃO}

Em estudo semelhante, autores [9] relataram decréscimo das frequiências cardíaca e respiratória e da pressão arterial média após administração epidural de xilazina em cães. Apesar da xilazina por via epidural apresentar efeitos sistêmicos menos evidentes que por via parenteral [21], neste estudo, observouse que a aplicação epidural desse fármaco também determinou efeitos depressores no sistema cardiovascular.

Nesta pesquisa, embora tenha sido observada redução da frequiência cardíaca, alterações sistêmicas mais graves, inerentes aos efeitos dos fármacos á2-agonistas, como arritmias, não foram identificadas. Deve-se salientar que a bradicardia pode representar efeito adverso importante quando está se realizando cirurgia em animais debilitados, portanto, a xilazina na anestesia epidural deve ser utilizada com cautela nesses animais. Neste estudo, os animais apresentaram redução na frequiência cardíaca (174 bpm até $120 \mathrm{bpm}$ ), voltando a subir para $142 \mathrm{bpm}$ até o final da mensuração dos parâmetros avaliados.

Utilizando-se lidocaína e xilazina para anestesia epidural em cães, não foram encontradas alte- 
Tabela 1. Médias da freqüência cardíaca, freqüência respiratória, temperatura retal e saturação de oxigênio de cutias (Dasyprocta prymnolopha) anestesiadas com anestesia epidural de xilazina e lidocaína, em experimento realizado no Hospital Veterinário da Universidade Federal do Piauí entre os meses de agosto e dezembro de 2006.

\begin{tabular}{|c|c|c|c|c|}
\hline Momentos & $\begin{array}{c}\text { Freqüência } \\
\text { Cardíaca }\end{array}$ & $\begin{array}{c}\text { Freqüência } \\
\text { Respiratória }\end{array}$ & $\begin{array}{c}\text { Temperatura } \\
\text { Retal }\end{array}$ & $\begin{array}{c}\text { Saturação } \\
\text { de Oxigênio }\end{array}$ \\
\hline 0 & $174,83 \mathrm{a}$ & $100,00 \mathrm{a}$ & $38,80 \mathrm{a}$ & $94,90 \mathrm{a}$ \\
\hline 1 & $165,83 \mathrm{a}$ & $56,00 \mathrm{~b}$ & $38,62 \mathrm{a}$ & $95,99 \mathrm{~b}$ \\
\hline 2 & $161,17 \mathrm{ba}$ & $33,33 \mathrm{c}$ & $38,47 \mathrm{a}$ & $95,33 \mathrm{c}$ \\
\hline 3 & $152,00 \mathrm{ba}$ & $30,00 \mathrm{c}$ & $38,35 \mathrm{ba}$ & $95,48 \mathrm{c}$ \\
\hline 4 & $153,67 \mathrm{ba}$ & $32,67 \mathrm{c}$ & $37,73 \mathrm{bac}$ & $95,86 \mathrm{c}$ \\
\hline 5 & $139,17 \mathrm{ba}$ & $28,00 \mathrm{c}$ & $37,20 \mathrm{bc}$ & $96,19 \mathrm{c}$ \\
\hline 6 & $120,83 \mathrm{~b}$ & $29,33 \mathrm{c}$ & $36,88 \mathrm{c}$ & $96,02 \mathrm{c}$ \\
\hline 7 & $142,17 \mathrm{ba}$ & $29,33 \mathrm{c}$ & $36,65 \mathrm{c}$ & $95,62 \mathrm{c}$ \\
\hline 8 & $142,17 \mathrm{ba}$ & $29,33 \mathrm{c}$ & $36,65 \mathrm{c}$ & $96,01 \mathrm{c}$ \\
\hline Média & $\mathbf{1 5 0 , 2 0 4 4}$ & $\mathbf{4 0 , 8 8 9}$ & $\mathbf{3 7 , 7 0 5 6}$ & $\mathbf{9 5 , 7 1}$ \\
\hline
\end{tabular}

Valores seguidos de letras diferentes na mesma coluna diferem entre si pelo teste de SNK a $\mathrm{p}<0,05$ para $\mathrm{p}$ mesmo parâmetro.

rações nos parametros respiratórios [6], entretanto, em outro ensaio semelhante pôde-se observar bradipnéia nos animais estudados [9], fato semelhante ao ocorrido às cutias utilizadas neste experimento.

A bradpnéia ocorreu logo após a aplicação da neuroleptoanalgesia. Há relatos de que uma das maiores desvantagens dos opióides agonistas, como o fentanil, é sua tendência em produzir depressão respiratória [21]. Outro fator contribuinte para a diminuição da frequiência respiratória no decorrer da anestesia é a ação a2 adrenoceptora agonista da xilazina que pode levar a bradipnéia [17].

Em estudos em cães, foi demonstrado que a administração epidural de $0,251 \mathrm{mg} / \mathrm{kg}$ de xilazina associada à lidocaína na anestesia epidural [6] produz efeito sedativo moderado, esses resultados divergem dos encontrados nesta pesquisa, na qual a utilização de uma dose duas vezes maior provocou uma sedação acentuada com insensibilidade nos membros pélvicos e torácicos.
Quando da utilização de xilazina associada à lidocaína por via epidural em cães, foi observado que esta associação produzia anestesia na região retroumbilical [6], fato semelhante ao encontrado nesta pesquisa em cutias.

A temperatura retal diminuiu desde o início da anestesia (M1) até o ultimo momento de avaliação deste parâmetro (sessenta minutos após a aplicação da anestesia epidural - M8). A redução foi em média $1,97 \pm 0,88$ graus, estando de acordo com o já descrito para esta espécie, após anestesia $[1,13,15,16]$. Este fato deve-se, provavelmente, ao pequeno tamanho destes animais, que perdem calor devido à pequena quantidade de tecido adiposo e também pela inibição de mecanismos termorreguladores centrais e periféricos [16]. Utilizando-se xilazina em coelhos [17] e em capivaras [4] também foi observada redução na temperatura.

A redução da temperatura pode também ser atribuída a exposição do animal a uma sala operatória com temperatura baixa [10]. 
O tempo hábil de anestesia de 51,66 \pm 4,68 minutos encontra-se abaixo da faixa de variação de $80,86+16,1$ quando utilizou-se apenas lidocaína na anestesia epidural também em cutias [15], essa diferença no retorno dos sinais pode ser explicada pela medicação pré-anestésica utilizada nos animais anestesiados apenas com lidocaína, que proporcionou uma maior sedação antes do início da anestesia epidural. Em cães, o tempo hábil de anestesia varia de 60 a 120 minutos [7] e, em ratos, 30 minutos [11].

$\mathrm{O}$ efeito analgésico da xilazina é conferido pela ativação dos alfa2-adrenoceptores na medula espinhal, a qual confere período de ação prolongado de analgesia [2].

A utilização de fentanil associado ao droperidol nas doses utilizadas não foi suficiente para produzir sedação nos animais a ponto de permitir a anestesia epidural com tranqüilidade, sendo então necessários mais estudos a respeito do uso da neuroleptoanalgesia nessa espécie.

\section{CONCLUSÕES}

Pode-se concluir que a utilização de xilazina/ lidocaína por via epidural, administrada no espaço lombossacro, produz anestesia na região retroumbilical, causando bloqueio anestésico mais cranial, o que favoreceria a realização de cirurgias na região pré-umbilical.

\section{NOTAS INFORMATIVAS}

${ }^{1}$ FENTANEST 0,05 mg/ml, Laboratório Cristália - São Paulo, Brasil.

${ }^{2}$ DROPERDAL 2,5mg/ml, Laboratório Cristália - São Paulo, Brasil.

${ }^{3}$ Wirath Industria e Comércio Ltda.

${ }^{4}$ LIDOSTON 2\%, Laboratório Ariston - São Paulo, Brasil. ${ }^{5}$ ROMPUM, Bayer S. A .Saúde Animal - São Paulo, Brasil.

\section{REFERÊNCIAS}

1 Araujo R.W., Feitosa Júnior F.S. \& Fernandes R.M. 2005. Efeitos cardiorespiratórios da associação tiletamina-zolazepam comparada com a associação tiletamina-zolazepam, atropina, xilazina através da extrapolação alométrica em cutias (Dasyprocta sp.). In: Resumos do IX Congresso da Associação Brasileira de Veterinários de Animais Selvagem (São José do Rio Preto). pp. 60.

2 Branson K.R., Ko J.C., Tranquilli W.J., Benson J. \& Thurmon J.C. 1993. Duration of analgesia induced by epidurally administered morphine and medetomidine in dogs. Journal of Veterinary Pharmacology and Therapeutics. 16: 369-372.

3 Carrol G.L. 1998. Stress related hormonal and metabolic responses to restraint with and without butorphanol admistration in pre conditioned goats. Laboratory Animal Science. 48: 387-390.

4 Cruz M.L., Luna S.P.L., Moura C. A ., Castro G.B., Neto F.J.T. \& Nishida S.M. 1998. Técnicas Anestésicas Injetáveis em Capivaras (Hydrochoerus hydrochaeris, Linné). Ciência Rural. 28: 411-415.

5 Cruz M.L., Luna S.P.L., Clark R.M.O., Castro G.B. \& Massone F. 1997. Epidural Anaesthesia using Lidocaine, Bupivacaine and a mixture of Lidocaine and Bupivacaine in Dogs. Journal of Veterinary Anaesthesia. 24: 30-32.

6 Gasparini S.S., Luna S.P.L., Cassu R.N., Uiechi E. \& Crocci A.J. 2007. Anestesia epidural com ropivacaína, lidocaína ou associação de lidocaína e xilazina em cães. Efeitos cardiorrespiratório e analgésico. Ciencia Rural. 37: 418-424.

7 Intelizano T. R., Dos Santos P.R., Futema F., Otsuki D.A. \& De Almeida T. I. 2002. Técnicas de anestesia local. In: Fantoni D. T. \& Cortopassi S. R. G. Anestesia em cães e gatos. São Paulo: Roca, pp. 199-208.

8 Ishiy H.M., Luna S.P.L., Gonçalves R.C. \& Cruz M.L. 2002. Uso da lidocaína isolada ou associada à quetamina ou ao butorfanbol, em anestesia epidural em cadelas, submetidas à ovariosalpingohisterectomia. Revista Brasileira de Ciência Veterinária. 9: 125-127.

9 Kelawala N.H., Kumar A., Chaudary S., Singh H.N. \& Singh H.P. 1996. Effects of epidural xylazine with diazepam premedication in dogs. Indian Veterinary Journal. 73: 552-557.

10 Little R.A. 1990. Ambient temperature and postoperative catabolism. Intensive Care Medicine. 16: 283-284.

11 Massone F. 2003. Anestesiologia Veterinária. 4. ed. Rio de Janeiro: Guanabara Koogan, 225p.

12 Mcmurphy R.M. 1993. Postoperative epidural analgesia. Veterinary Clinics of North America: Small Animal Practice. 23: 703-716.

13 Moura S.G., Fernandes R.M., Silva Júnior J.R., Barradas A.L.B., Araújo W.R., Carvalho M.A.M. \& Lopes J.B. 2002. Cloridrato de tiletamina associado com cloridrato de zolazepam na tranquilização e anestesia de cutias (Dasyprocta sp). In: Resumos do VI Congresso e XI Encontro da Associação Brasileira de Veterinários de Animais Selvagens (Guarapari, Brasil). pp. 70 . 
14 O'Hair K.C., Dood K.T., Phillips Y.Y. \& Beattie R.J. 1998. Cardiopulmonary effects of nalbuphine and hidrochloride and butorphanol tartarate in sheep. Laboratory Animal Science. 38: 58-61.

15 Oliveira F.S., Martins L.L., Duque J.C., Pauloni A.P. \& Valadão C.A.A. 2006. Anestesia epidural em cutias (Dasyprocta azarae). Acta Scientiae Veterinariae. 34: 89-91.

16 Pachaly J.R. 1999. Contenção da cutia, Dasyprota azarae Lichtenstein, 1823 (rodentia; mammalia), pela associação de cetamina, cloridrato de xilazina e sulfato de atropina - definição de protocolos posológicos individuais com base em extrapolação alométrica interespecífica, Arquivos de Ciências Veterinárias e Zoologia da Unipar. 2: 83-84.

17 Popilskis S.J., Oz M.C., Gorman P., Florestal A. \& Kohn D.F. 1991. Comparison Of Xylazine With Tiletamine-Zolazepam (Telazol)And Xylazine-Ketamine Anesthesia Ir Rabbits. Laboratory Animal Science. 41: 51-53.

18 Rector E., Otto K., Kietzmann M., Kramer S., Landwehr S., Hart S. \& Nolte I. 1997. Evaluation of the antinociceptive effect of xylazine after epidural administration in dogs under general anesthesia with isoflurane. Berliner und Munchener tierarztliche Wochenschrift. 110: 5-23.

19 SAS Institute. 1997. SAS/STAT software: changes and enhancements through release 6.12. Cary: Statistical Analysis System Institute. $1167 \mathrm{p}$.

20 Vieira R.J., Meira C., Quessada A.M., Facin P. R., Massone F., Fuck E. \& Curi P.R. 1993. Xilazina e lidocaina: anestesia peridural comparativa em bovinos. Veterinária e Zootecnia. 5: 105-112.

21 Waterman A.E., Linvingston A. \& Amin A. 1991. Analgesic activity and respiratory effects of butorphanol in sheep. Research and Veterinary Science. 51: 19-23.

22 Weissman C. 1990. The metabolic response to stress: An overview and update. Anesthesiology. 73: 308-327. 